\title{
Energy Collection by a Polymer Shish Kabob
}

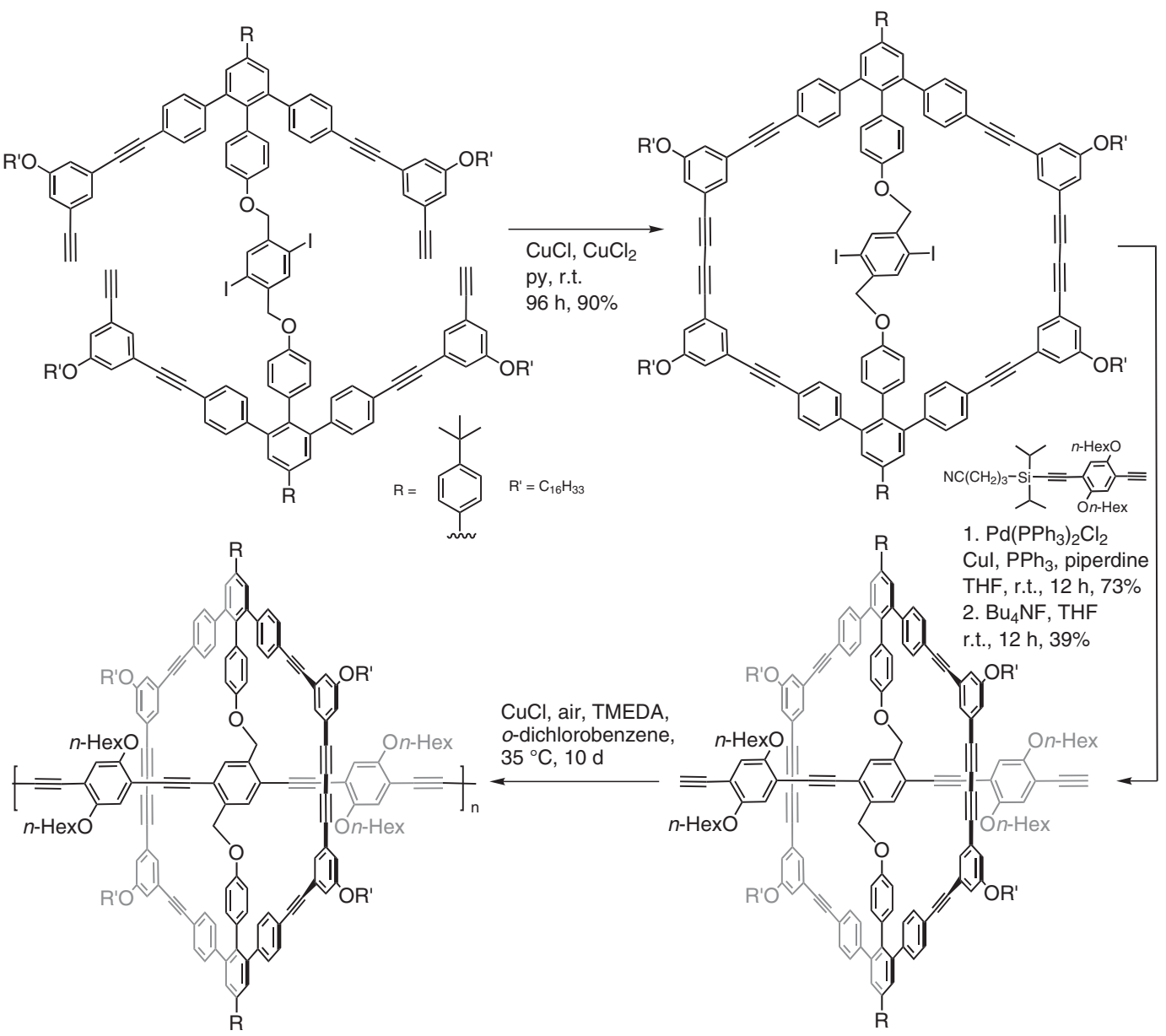

Synthesis of

Materials and

Unnatural Products

Key words

polymers

energy transfer

macrocycles

Significance: Future energy and photonic technologies will rely on the delivery of electronic excitations to a specific location. In addition to synthesizing an impressive structure, the authors demonstrate the use of geometrically fixed independent chromophores to create systems capable of having two excitations present at the same time. This leads to the annihilation of the excitations when they combine. Delivering multiple quanta of energy to reaction centers has long been of interest in the search for new photochemistry and physics.
Comment: The authors demonstrate that the collection of multiple photons is dependent on the molecular weight and that longer polymers can accumulate multiple excitations at lower laser pump powers than the shorter polymers. The molecular architecture is complex and is not likely to be widely emulated. However, this report will serve to guide others to develop more practical energy collection systems.

SYNFACTS Contributors: Timothy M. Swager

Synfacts 2007, 10, 1041-1041 Published online: 20.09.2007

Dol: 10.1055/s-2007-968994; Reg-No.: S10007SF 